Europhysics Letters

PREPRINT

\title{
Motion of vortices implies chaos in Bohmian mechanics
}

\author{
D. A. Wisniacki ${ }^{1}$ and F. E. R. Pujals ${ }^{2,3}$ \\ 1 Departamento de Física "J.J. Giambiagi", FCEN, UBA, Pabellón 1, Ciudad Univer- \\ sitaria, 1428 Buenos Aires, Argentina. \\ 2 Department of Mathematics, University of Toronto, Toronto, Ontario, Canada M5S \\ $3 G 3$. \\ 3 IMPA-OS, Dona Castorina 110, 22460-320 Rio de Janeiro, Brasil.
}

PACS. 03.65.-w - Quantum mechanics.

PACS. 03.65.Ta - Foundations of quantum mechanics; measurement theory.

PACS. 05.45.Mt - Quantum chaos; semiclassical methods.

\begin{abstract}
Bohmian mechanics is a causal interpretation of quantum mechanics in which particles describe trajectories guided by the wave function. The dynamics in the vicinity of nodes of the wave function, usually called vortices, is regular if they are at rest. However, vortices generically move during time evolution of the system. We show that this movement is the origin of chaotic behavior of quantum trajectories. As an example, our general result is illustrated numerically in the two-dimensional isotropic harmonic oscillator.
\end{abstract}

De Broglie-Bohm's (BB) approach to quantum mechanics has experienced an increased popularity in recent years. This is due to the fact that it combines the accuracy of the standard quantum description with the intuitive insight derived from the causal trajectory formalism, thus providing a powerful theoretical tool to understand the physical mechanisms underlying microscopic phenomena [1,2]. Although the behavior of quantum trajectories is very different from classical solutions it can be used to gain intuition in many physical phenomena. Numerous examples can be found in different areas of research. In particular, we can mention studies of barrier tunneling in smooth potentials [3], the quantum back-reaction problem [4] and ballistic transport of electrons in nanowires [5].

According to the BB theory of quantum motion, a particle moves in a deterministic orbit under the influence of the external potential and a quantum potential generated by the wave function. This quantum potential can be very intricate because it encodes information on wave interferences. Based on it, Bohm already predicted complex behavior of the quantum trajectories in his seminal work [6]. This was recently confirmed in several studies when presence of chaos in various systems has been shown numerically [7-9]. However, the mechanisms that cause such a complex behavior is still lacking. In this letter we show that movement of the zeros of the wave function, commonly known as vortices, implies chaos in the dynamics of quantum trajectories. Such a movement perturbs the velocity field producing transverse homoclinic orbits that generate the well known Smale horseshoes which is the origin of complex behavior. Our assertion is based on an analytical proof in a simplified model which resembled the velocity field near the vortices. In addition, we present a numerical study in a 2-D

(C) EDP Sciences 
isotropic harmonic oscillator that displays a route to chaos dominated by this mechanism. It is important to mention that there is no agreement in previous works about the influence of vortices on the chaotic motion of quantum trajectories [8-11].

The fundamental equations in the BB theory are derived from the introduction of the wave function in polar form, $\psi(\mathbf{r}, t)=R(\mathbf{r}, t) \mathrm{e}^{\mathrm{i} S(\mathbf{r}, t)}$ (throughout the paper $\hbar$ is set equal to 1 ), into the time-dependent Schrödinger equation, thus obtaining two real equations:

$$
\begin{gathered}
\frac{\partial R^{2}}{\partial t}+\nabla \cdot\left(R^{2} \frac{\nabla S}{m}\right)=0, \\
\frac{\partial S}{\partial t}+\frac{(\nabla S)^{2}}{2 m}+V-\frac{1}{2 m} \frac{\nabla^{2} R}{R}=0,
\end{gathered}
$$

which are the continuity and quantum Hamilton-Jacobi equations, respectively. The last term in the left-hand-side of Eq. (2) is the so-called quantum potential, a non-local function determined by the quantum state, which, together with $V$, determines the total force acting on the system. Similarly to what happens in the usual classical Hamilton-Jacobi theory, quantum trajectories of a particle of mass $m$ can then be defined by means of the following velocity field equation:

$$
\mathbf{v}=\dot{\mathbf{r}}=\frac{1}{m} \nabla S=\frac{i}{2 m} \frac{\psi \nabla \psi^{*}-\psi^{*} \nabla \psi}{|\psi|^{2}} .
$$

Vortices appear naturally in the BB framework. They result from wave function interferences so they have no classical explanation. In systems without magnetic field, the bulk vorticity $\nabla \times \mathbf{v}$ in the probability fluid is determined by the points where the phase $S$ is singular. This may occur only at points where the wave function vanishes. This condition is fulfilled by isolated points in a 2-D system and lines in a 3-D system. Due to the single-valuedness of the wave function, the circulation $\Gamma$ along any closed contour $\xi$ encircling a vortex must be quantized, that is,

$$
\Gamma=\int_{\xi} \dot{\mathbf{r}} d \mathbf{r}=\frac{2 \pi n}{m},
$$

with $n$ an integer $[12,13]$. So, the velocity $\mathbf{v}$ must diverge as one approaches a vortex. In fact, the time dependent velocity field in the vicinity of a vortex located at time $t$ in $\mathbf{r}_{\mathbf{v}}(t)$ is given by

$$
\mathbf{v}=\frac{-i}{2 m} \frac{\left[\mathbf{r}-\mathbf{r}_{\mathbf{v}}(t)\right] \times \mathbf{w} \times \mathbf{w}^{*}}{\left|\left[\mathbf{r}-\mathbf{r}_{\mathbf{v}}(t)\right] \cdot \mathbf{w}\right|^{2}},
$$

where $\mathbf{w} \equiv \nabla \psi\left(\mathbf{r}_{\mathbf{v}}(t)\right)[10,14]$. We consider here 2-D systems but we belive that our results are valid for systems of higher dimensions.

Before presenting our analytical results we show a numerical simulation of quantum trajectories in a system consisting of a particle of unit mass in a 2-D isotropic harmonic oscillator. We have set the angular frequency $\omega=1$, so the Hamiltonian of the system results

$$
H=-\frac{1}{2}\left(\frac{\partial^{2}}{\partial x^{2}}+\frac{\partial^{2}}{\partial y^{2}}\right)+\frac{1}{2}\left(x^{2}+y^{2}\right) .
$$

The eigenenergies are $E_{n_{x} n_{y}}=n_{x}+n_{y}+1$ and the eigenfunctions $\phi_{n_{x} n_{y}}(x, y)=\exp \left(-\frac{1}{2}\left(x^{2}+\right.\right.$ $\left.\left.y^{2}\right)\right) H_{n_{x}}(x) H_{n_{y}}(y) / \sqrt{\pi 2^{n_{x}+n_{y}} n_{x} ! n_{y} !}$ with $n_{x}=0,1, \ldots, n_{y}=0,1, \ldots$ where $H_{n}$ is the n-th degree Hermite polynomial. 


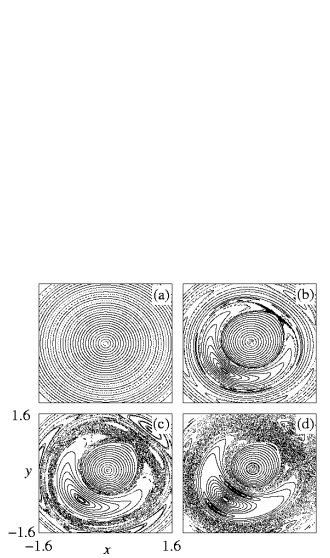

Fig. 1

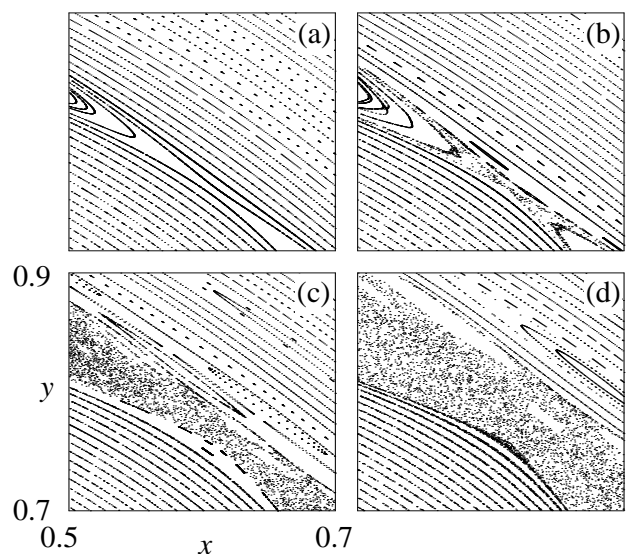

Fig. 2

Fig. 1 - Poincare surface of section for the quantum trajectories generated by the wave function of Eq. (7) with $b=c$ and $a / b=0$ (a), 0.0553 (b), 0.1138 (c) and 0.17651 (d) with fixed value of $\gamma_{1}=3.876968$ and $\gamma_{2}=2.684916$. The trajectories of the vortex of the corresponding wave functions are shown in Fig. 3

Fig. 2 - Part of the Poincare surface of section for the quantum trajectories generated by the wave function of Eq. (7) with small values of $a / b$. The parameter $b=c$ and $a / b=0.01082$ (a), 0.02175 (b), 0.0328 (c) and 0.0440 (d) with fixed value of $\gamma_{1}=3.876968$ and $\gamma_{2}=2.684916$.

We have chosen the following general combination of the first three eigenstates of the Hamiltonian of Eq. ([6) as initial state

$$
\psi_{0}=a \phi_{00}+b \exp \left(-i \gamma_{1}\right) \phi_{10}+c \exp \left(-i \gamma_{2}\right) \phi_{01},
$$

with $a, b, c, \gamma_{1}$ and $\gamma_{2}$ real numbers and $a^{2}+b^{2}+c^{2}=1$ (the normalization condition). A remarkable point is that this state generates a periodic time dependent velocity field with only one vortex. Moreover, the trajectory of the vortex can be obtained analytically resulting

$$
\mathbf{r}_{\mathbf{v}}(t)=\left(x_{v}(t), y_{v}(t)\right)=\left(\frac{a}{\sqrt{2} b} \frac{\sin \left(\gamma_{2}-t\right)}{\sin \left(\gamma_{1}-\gamma_{2}\right)}, \frac{a}{\sqrt{2} c} \frac{\sin \left(\gamma_{1}-t\right)}{\sin \left(\gamma_{1}-\gamma_{2}\right)}\right) .
$$

This fact allows us to see the influence of the movement of a vortex in the dynamics of the quantum trajectories, without taking into account the possibility of instantaneous creation or annihilation of a vortex pair with opposite circulation $[14,15]$. This important phenomenon will be studied elsewhere [16].

The non-autonomous velocity field generated by the wave function of Eq. (7) is periodic so the best surface of section is given by fixing $t=2 \pi n$ with $n=0,1, \ldots$ (also called a stroboscopic view). Fig. 1 1 shows surfaces of section with $b=c$ and $a / b=0,0.0553,0.1138$ and 0.17651 with fixed value of $\gamma_{1}=3.876968$ and $\gamma_{2}=2.684916$. The trajectories of the vortex for the cases studied in Fig. 1 are plotted in Fig. 3 A clear transition to chaos appears as the parameter $a / b$ is increased. If the position of vortex is fixed the trajectories are regular and no chaos is present [see Fig. 1(a)] . However, irregular dynamics is observed for small $a / b$ [Fig. 1(b)]. The transition to irregular dynamics is shown in Fig. 2 The movement of the vortex produces a saddle point near $(0.6,0.75)$ and their stable and unstable manifolds have a topological transverse intersection generating the well known homoclinic tangle [17]. 


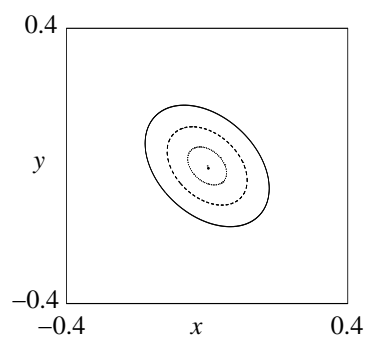

Fig. 3 - Path described by the vortex [Eq. [8] of the velocity field generated by wave functions of Eq. (7) with $b=c$ and $a / b=0$ (filled circle), 0.0553 (dotted line), 0.1138 (dashed line) and 0.17651 (solid line) with fixed value of $\gamma_{1}=3.876968$ and $\gamma_{2}=2.684916$.

Now we will show analytical results that explain the numerical experiments presented before. Our starting point is the following model: a particle of unit mass on the plane in the velocity field of Eq. (5.) with the constraints that the trajectory of the vortex is a time periodic curve and $w_{x}=i w_{y}[18]$. Thus, the non-autonomous vector field is equal to

$$
\begin{aligned}
& v_{x}=\frac{-\left(y-y_{v}(t)\right)}{\left(x-x_{v}(t)\right)^{2}+\left(y-y_{v}(t)\right)^{2}} \\
& v_{y}=\frac{\left(x-x_{v}(t)\right)}{\left(x-x_{v}(t)\right)^{2}+\left(y-y_{y}(t)\right)^{2}} .
\end{aligned}
$$

Taking $\bar{x}=x-x_{v}(t) \bar{y}=y-y_{v}(t)$ and writing in polar coordinates $(\bar{x}=r \cos (\theta), \bar{y}=$ $r \sin (\theta))$, the velocity field of Eq. (9) results

$$
\begin{aligned}
& v_{r}=r\left[\sin (\theta) y_{v}(t)+x_{v}(t) \cos (\theta)\right] \\
& v_{\theta}=\frac{1}{r}+\cos (\theta) y_{v}(t)-x_{v}(t) \sin (\theta) .
\end{aligned}
$$

This non-autonomous velocity field can be seen as a perturbation of the autonomous velocity field $\mathbf{v}_{0} \equiv\left(0, \frac{1}{r}\right)$, with $\mathbf{G}(r, \theta, t) \equiv\left(r\left[\sin (\theta) y_{v}(t)+x_{v}(t) \cos (\theta)\right], \cos (\theta) y_{v}(t)-x_{v}(t) \sin (\theta)\right)$ the time-periodic perturbation. Note that the field is induced by the time dependent Hamiltonian

$$
H(r, \theta, t)=\frac{1}{2} \log (r)+r\left[\cos (\theta) y_{v}(t)-\sin (\theta) x_{v}(t)\right] .
$$

We consider periodic curves $\mathbf{r}_{\mathbf{v}}(t)$ such that

$$
\int_{0}^{T_{0}} \cos (\theta) y_{v}(t)-x_{v}(t) \sin (\theta) d t d s \neq 0 .
$$

Under these hypothesis the following property can be proved:

There exists a saddle periodic orbit of the flow associated to the vector field of Eq. (9), exhibiting a homoclinic transversal intersection.

This result, which implies that quantum trajectories show topological chaos, is the main finding of our work. We illustrate here the geometrical arguments of the proof and we leave the full details for a future publication [19]. We notice that a periodic orbit of saddle type 

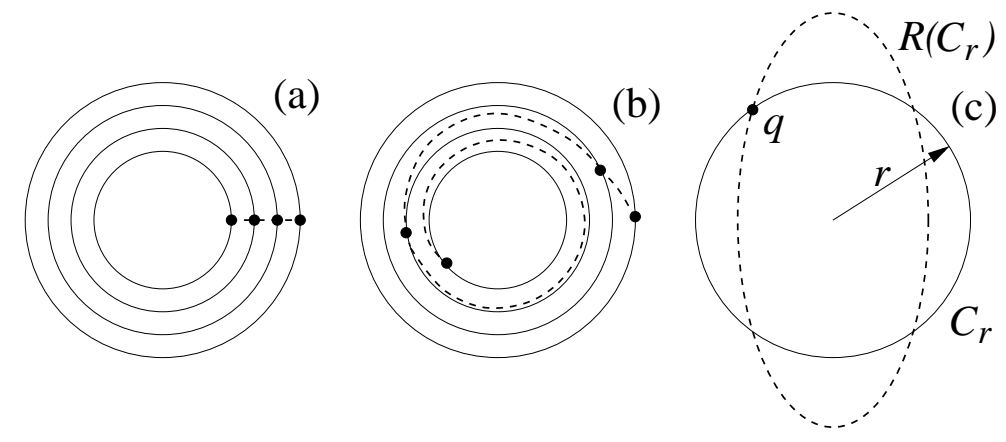

Fig. 4 - (a) Schematic plot of the invariants of the non-perturbed map $R^{0}$ generated by the autonomous velocity field $\mathbf{v}_{0}$ (solid lines). With dashed line it is plotted a segment with $\theta=0$. (b) Non-perturbed mapped $R^{0}$ of the segment with $\theta=0$ (dashed line). (c) It is showed with dashed line the image of the perturbed map $R$ of a circle $C_{r}$ with radius $r$ (solid line). A point $q \in R\left(C_{r}\right) \cap C_{r}$ is also plotted.

exhibits a homoclinic transversal intersection if their stable and unstable manifolds intersect each other and the tangents of the manifolds are not colinear at the intersection. Transversal homoclinic intersections (or homoclinic tangles) beat at the heart of chaos. This is because in the region of a homoclinic tangle, initial conditions are subject to a violent stretching and folding process, the two essential ingredients for chaotic behavior.

Let us start to show the main result of the letter considering some important charateristics and properties of the flows generated by the velocity fields $\mathbf{v}_{0}$ and $\mathbf{v}$. The flow $\Phi_{t}^{0}$ associated with the autonomous velocity field $\mathbf{v}_{0}$ is defined for every $(\bar{x}, \bar{y}) \neq(0,0)$. Thus, given a positive time $T_{0}$, the map $R^{0}=\Phi_{T_{0}}^{0}: \Re^{2} \backslash\{(0,0)\} \rightarrow \Re^{2} \backslash\{(0,0)\}$ is well defined. It is straightforward to show that the map $R^{0}$ written in polar coordinates results $R^{0}=\left(R_{r}^{0}(r, \theta), R_{\theta}^{0}(r, \theta)\right)=$ $\left(r, \theta+\frac{1}{r} T_{0}\right)$. Map $R^{0}$ keeps invariant the set of points with same radius; i.e., it keeps invariant the circles. Moreover, the circles are rotated with a rate of rotation inversely proportional

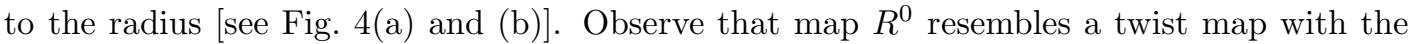
difference that in the present case, the rate of rotation grows to infinity when the radius is reduced. In this respect, it is important to mention that for generic conservative perturbations of the twist map the existence of homoclinic points associated to a saddle periodic point was proved [20] . This result was also extended to time periodic perturbations of a flow which exhibit an elliptic singularity [21].

We have assumed that the vortex moves periodically along a curve, that is, $\mathbf{r}_{\mathbf{v}}(t)=\mathbf{r}_{\mathbf{v}}(t+$ $\left.T_{0}\right)$. Then, the time dependent velocity field $\mathbf{v}$ induces a flow $\Phi_{t}$ which is defined for every $(\bar{x}, \bar{y}) \neq\{(0,0)\}$. So, the map $R=\Phi_{T_{0}}: \Re^{2} \backslash\{(0,0)\} \rightarrow \Re^{2} \backslash\{(0,0)\}$ is well defined. Note that flow $\Phi_{t}$ is generated by a time periodic Hamiltonian [Eq. [10] ], so $R$ is a conservative map. Also, $R$ can be extended continuously to $(0,0)$ defining $R(0,0) \equiv(0,0)$ [note that $R \rightarrow 0$ when $(\bar{x}, \bar{y}) \rightarrow(0,0)]$. Then, it follows that map $R$ verifies:

Property A: Like $R^{0}$, map $R$ also has the property that circles are rotated with a rate of rotation inversely proportional to the radius [see Fig. [4(a) and (b)]. In other words, if map $R$ is written in polar coordinates $R(r, \theta)=\left(R_{r}(r, \theta), R_{\theta}(r, \theta)\right)$, then $\partial_{r} R_{\theta}$ is of the order of $1 / r^{2}$.

Property B: The image of a small circle of radio $r$ intersects transversally this circle; i.e, $R\left(C_{r}\right) \cap C_{r} \neq \emptyset$ and if $q \in R\left(C_{r}\right) \cap C_{r}$ then the tangent to $R\left(C_{r}\right)$ and to $C_{r}$ in $q$ are not collinear [see Fig. 迎(c)].

From properties $A$ and $B$ of the perturbed map $R$ it follows that for arbitrarily small $r$ the map $R$ has a fixed point $p_{0}$ with radius smaller than $r$ exhibiting homoclinic transversal 
EUROPHYSICS LETTERS

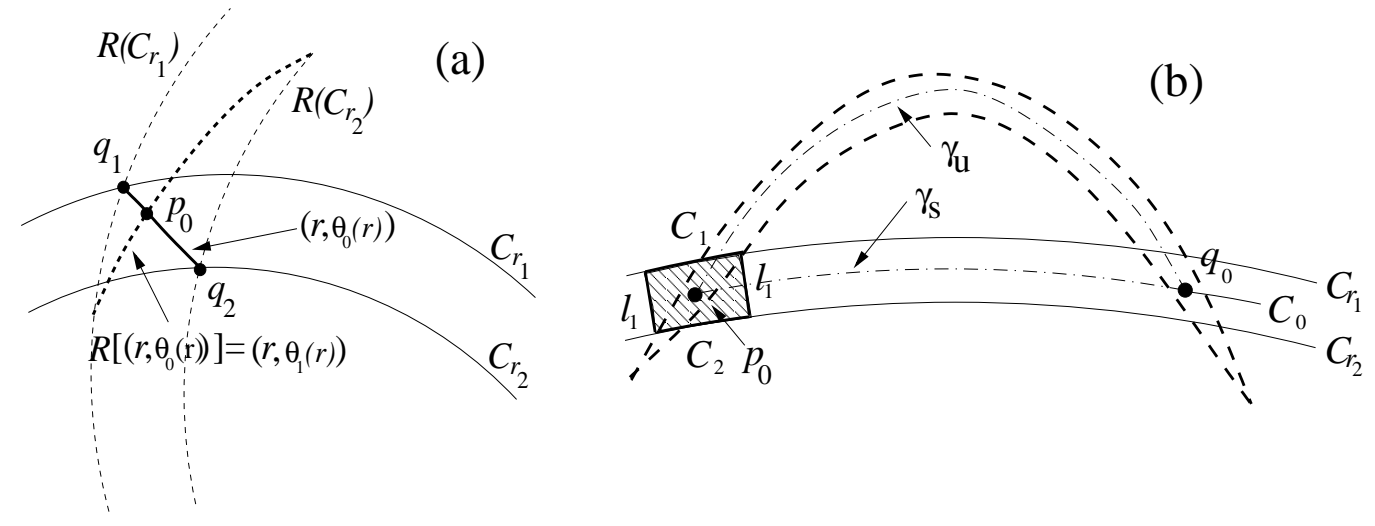

Fig. 5 - Schematic plots to show the existence of a saddle periodic point $p_{0}$ of the map $R$ with a homoclinic transversal intersection. (a) The curve $r \rightarrow\left(r, \theta_{0}(r)\right)$ is plotted with thick solid line, and the curve $r \rightarrow R\left(\left(r, \theta_{0}(r)\right)\right)=\left(r, \theta_{1}(r)\right)$ is plotted with thick dashed line. Two invariants circles $\left(C_{r_{1}}\right.$ and $C_{r_{2}}$ ) and their perturbed mapping are also plotted. Note the transversal intersection between the circles an their respective mapping (points $q_{1}$ and $q_{2}$ ). (b) The rectangle $B$ (shaded area) is bounded by two pieces of arcs $C_{1}$ and $C_{2}$ contained in $C_{r_{1}}$ and $C_{r_{2}}$ (where $r_{1}$ and $r_{2}$ are closed to $r_{0}$ verifying $r_{1}<r_{0}<r_{2}$ ) and two segments $l_{1}$ and $l_{2}$ contained in two different rays of constant angle. The segments $\gamma_{u}$ and $\gamma_{s}$, that connects the periodic point $p_{0}$ with the transversal intersection $q_{0}$, are ploted with dash-dotted lines. The mapping $R(B)$ of the considered rectangle is also ploted with thick dashed line.

point. Of course, this result implies that the vector field of Eq. (9) has a saddle periodic orbit with an homoclinic transversal intersection.

We show the previous result in two steps. First, let us display that the perturbed map $R$ has a fixed point. Property $B$ guarantees the existence of the curve $\left(r, \theta_{0}(r)\right)$ plotted in Fig. [5 (a). Note that $\theta_{0}(r)$ is the angular coordinate of a point on the intersection of a circle $C_{r}$ with its image $R\left(C_{r}\right)$. A point on such a curve is mapped to other point with the same radius $r$ but with a different angle $\theta_{1}(r)$,

$$
R\left(r, \theta_{0}(r)\right)=\left(R_{r}\left(r, \theta_{0}(r)\right), R_{\theta}\left(r, \theta_{0}(r)\right)=\left(r, \theta_{1}(r)\right) .\right.
$$

It is clear that map $R$ has a fixed point if there exist an $r_{0}$ such that $\theta_{0}\left(r_{0}\right)=\theta_{1}\left(r_{0}\right)$. In other words, curves $\left(r, \theta_{0}(r)\right)$ and $\left(r, \theta_{1}(r)\right)$ of Fig. 5 (a) intersect at $r_{0}$. From property $A$ follows that the variation of $\theta_{1}(r)$ is larger than the variation of $\theta_{0}(r)$; in fact, the derivative of $\theta_{1}(r)$ is of the order of $1 / r^{2}$. This fact guarantees the existence of $r_{0}$ in the vicinity of $r \rightarrow 0$ [19].

Now we will see that the mentioned fixed point has a transversal homoclinic intersection of their stable and unstable manifolds. We recall that the stable manifold is the set of points that converges to the fixed point by forward iteration of the dynamic. Conversely, the points on the unstable manifold converge to the fixed point by backward iteration. In Fig. [5) we consider a circle $C_{0}$ of radius $r_{0}$ containing the fixed point $p_{0}$. Due to property $B, C_{0}$ and its image $R\left(C_{0}\right)$ has al least an additional point of intersection denoted by $q_{0}$. Points $p_{0}$ and $q_{0}$ are connected by the segments $\gamma_{s}$ and $\gamma_{u}$ of $C_{0}$ and $R\left(C_{0}\right)$ respectively. Let us consider

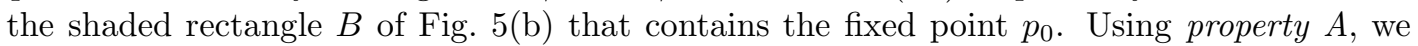
have deduced that segments of constant angle $l_{1}$ and $l_{2}$ are stretched by $R$, and this shows that rectangle $B$ is contracted by $R$ along directions close to the tangent of the circles $C_{r}$ and expanded along vectors close to the tangent of $R\left(C_{r}\right)$. This is displayed in Fig. 5 b) where the mapping of the rectangle $B$ is plotted with thick dashed line. Moreover, we have proved 
in Ref. [19] that $R(B)$ is close to $\gamma_{u}$ and intersects $C_{0}$ in a point near $q_{0}$, and that, $R^{-1}(B)$ is close to $\gamma_{s}$ and intersects $C_{0}$ near $q_{0}$. This implies that the unstable manifold of $p_{0}$ is close to $\gamma_{u}$ and the stable manifold is close to $\gamma_{s}$. We recall that $\gamma_{u}$ cross $\gamma_{u}$ at $q_{0}$, then the stable and unstable manifolds of $p_{0}$ have a transversal intersection near $q_{0}$.

In summary, we have found an universal mechanism leading to quantum trajectories having chaotic behavior. We have shown that the movement of vortices is a generic time dependent perturbation of an autonomous velocity field which creates a saddle periodic orbit with a transversal homoclinic intersection of their stable and unstable manifolds. This transversal intersection generates the well known Smale horseshoe which is the origin of complexity . Our results should be useful due to the fact that such deterministic quantum orbits are an important theoretical tool for understanding and interpreting several processes in different fields. On the other hand, our geometrical analysis of a singular velocity field could be important for both theoretical and applied problems of dynamical systems, as for example advection in non-stationary fluids [22].

This work received financial support from CONICET and UBACYT (X248). We thank G. Lozano and F. Duffy for useful comments.

\section{REFERENCES}

[1] P. R. Holland, The Quantum Theory of Motion (Cambridge University Press, Cambridge) 1993.

[2] D. Dürr, S. Goldstein And N. Zanghì, J. Stat. Phys., 67 (1992) 843.

[3] C. L. Lopreore and R. E. Wyatt, Phys. Rev. Lett., 82 (1999) 5190.

[4] O. V. Prezhdo and C. Brooksby, Phys. Rev. Lett., 86 (2001) 3215.

[5] H. Wu And D. W. L. Sprung, Phys. Lett. A, 196 (1994) 229.

[6] D. Bohm, Phys. Rev., 85 (1952) 166; 85 (1952) 194.

[7] R. H. Parmenter and R. W. Valentine, Phys. Lett. A, 201 (1995) 1; S. Konkel And A. J. Malowski, Phys. Lett. A, 238 (1998) 95. J. A. Sales and J. Florencio, Phys. Rev. E, 67 (2003) 016216.

[8] H. Frisk, Phys. Lett. A, 227 (1997) 139;

[9] H. Wu And D. W. L. Sprung, Phys. Lett. A, 261 (1999) 150;

[10] P. Falsaperla and G. Fonte, Phys. Lett. A, 316 (2003) 382.

[11] A. Valentini and H. Westman, Proc. R. Soc. A, 461 (2005) 253.

[12] P. A. M. Dirac, Proc. R. Soc. A, 133 (1931) 60.

[13] I. Bialynicki-Birula and Z. Bialynicki-Birula, Phys. Rev. D, 3 (1971) 2410.

[14] I. Bialynicki-Birula, Z. Bialynicki-Birula and C. Sliwa, Phys. Rev. A, 61 (2000) 5190.

[15] J. O. Hirschfelder, J. Chem. Phys., 67 (1978) 5477.

[16] D.A.Wisniacki, F. Borondo and E. PujAls, in preparation.

[17] J. Palis And F. TAKens, Hyperbolicity and sensitive chaotic dynamics at homoclinic bifurcations (Cambridge University Press, Cambridge) 1993.

[18] This is the simplest toy model because $w_{x}$ and $w_{y}$ are time dependent functions given by the derivatives of the wave function.

[19] E. Pujals and D. A Wisniacki, in preparation.

[20] E. Zehnder, Comm. Pure Appl. Math., 26 (1973) 131.

[21] J. Guckenheimer And Ph. Holmes, Nonlinear oscillations, dynamical systems, and bifurcations of vector fields (Springer-Verlag, New York) 1990

[22] L. Kuznetsov and G. M. Zaslavsky, Phys. Rev. E, 58 (1998) 7330; G. Boffeta, A. Celani and P. Franzese, J. Phys. A: Math. Gen., 29 (1996) 3749. 